\title{
Single cold atom as efficient stationary source of EPR-entangled light
}

\author{
David Vitali \\ CNISM and Dipartimento di Fisica, Università di Camerino, 62032 Camerino, Italy
}

Giovanna Morigi

Grup d'Optica, Departament de Fisica, Universitat Autonoma de Barcelona, 08193 Bellaterra, Spain

\author{
Jürgen Eschner \\ ICFO-Institut de Ciencies Fotoniques, Mediterranean Technology Park, 08860 Castelldefels (Barcelona), Spain
}

(Received 27 May 2006; published 20 November 2006)

\begin{abstract}
The Stokes and anti-Stokes components of the spectrum of resonance fluorescence of a single trapped atom, which originate from the mechanical coupling between the scattered photons and the quantized motion of the atomic center of mass, exhibit quantum correlations which are of two-mode-squeezing type. We study and demonstrate the build-up of such correlations in a specific setup, which is experimentally accessible, and where the atom acts as efficient and continuous source of EPR-entangled, two-mode squeezed light.
\end{abstract}

DOI: 10.1103/PhysRevA.74.053814 PACS number(s): 42.50.Dv, 03.67.Mn, 32.80.Qk, 32.80.Lg

\section{INTRODUCTION}

The control of atom-photon interaction is an object of intensive research for its potentialities in quantum networking. In fact, several experimental realizations have accessed regimes of engineering atom-photon interactions and have opened promising perspectives for implementing controlled nonlinear dynamics with simple quantum optical systems. Fundamental steps in this direction have been, amongst others, the generation of entangled light in atomic ensembles $[1,2]$, atomic memory for quantum states of light [3-7], and entanglement of remote ensembles [8-10]. At the single atom level, entanglement between a single atom and its emitted photon [11] has been demonstrated in Refs. [12,13], while in cavity quantum electrodynamics generation of quantum light has been achieved, such as lasing at the single atom level [14,15], controlled single-photon generation [16-19], as well as quantum state and entanglement engineering in the microwave regime [20].

Quantum networking with single trapped atoms or ions shows several advantages, due to the high degree of control one can achieve on these systems [19,21,22]. Control can be gained on the internal as well as on the external degrees of freedom, which can both be interfaced with light by exchange of angular and linear momentum. In particular, by coupling the atomic external degrees of freedom with photons via the mechanical effect of light, atom-photon interfaces for continuous variables can be implemented even at the level of a single atom [23-26]. This concept has been specifically applied in Refs. [25,26], where the realization of a pulsed optical parametric amplifier based on a single cold trapped atom inside a high-finesse optical cavity was proposed, and it was shown theoretically that this system allows for the controlled, quantum-coherent generation of entangled light pulses by exploiting the mechanical effects of atomphoton interaction.

In this paper we investigate the quantum correlations between the Stokes and anti-Stokes sidebands of the resonance fluorescence of a trapped atom, i.e., between the spectral components which are due to the coupling of the electromag- netic field to the atom's oscillatory motion [27-30]. The spectrum is studied for an atom tightly confined inside a resonator and continuously driven by a laser, in the setup sketched in Fig. 1. This setup has been considered in Refs. $[25,26]$ for the case of pulsed excitation, where scattering could be considered coherent. In the present work, the atom is continuously driven and hence both coherent and incoherent scattering processes determine the dynamics of the system. We find that in a suitable parameter regime the Stokes and anti-Stokes spectral components of the resonance fluorescence are two-mode squeezed, that is, their amplitude and phase quadratures are quantum correlated. In fact, the variance of the difference of the amplitude quadratures of the two sideband modes, as well as the variance of the sum of their phase quadratures, are squeezed below the shot noise

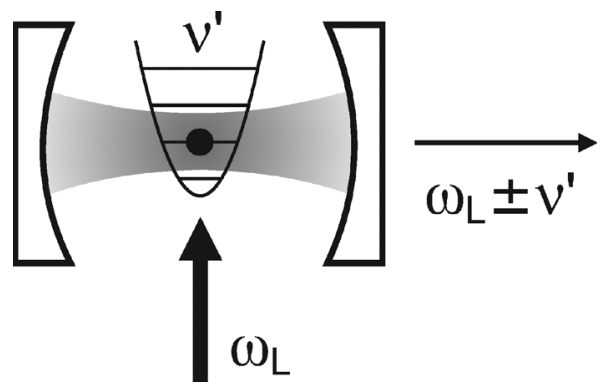

FIG. 1. Layout of the system. A single atom is confined by an external potential inside an optical cavity and is driven by a laser. The cavity is resonant with the motion-induced Stokes and antiStokes components of the resonance fluorescence. Correlations between these spectral component are measured in the cavity output. The orientation of the considered vibrational mode has nonzero projection onto the laser direction. A possible geometry to implement the system would be an $F=0$ to $F^{\prime}=1$ atomic transition with the quantization axis $\vec{B}$ along the cavity axis, and $\vec{B}$, laser wave vector, and laser polarization mutually orthogonal, and a motional mode parallel to the laser direction. More details can be found in Refs. [25,26], where pulsed coherent excitation was considered. In the present paper we deal with continuous laser excitation. 
limit, hence reproducing the salient properties of the entangled, simultaneous eigenstate of relative distance and total momentum of two particles, as considered in the original EPR paradox [31,32]. In our model, entanglement between the modes originates from the mechanical coupling of the electromagnetic field with the quantum motion of the atom, and it is endorsed by a specific setup, which achieves resonant emission of the Stokes and anti-Stokes photons. In this regime, the single atom acts as an efficient continuous source of EPR-entangled, two-mode squeezed light.

Conventionally, two-mode squeezed states emerge from the nonlinear optical interaction of a laser with a crystal, i.e., from parametric amplification or oscillation. As such, the phenomenon is the result of many-atom dynamics, often described by a simple nonlinear polarization model. In the single-atom case additional features appear which are due to the coherent microscopic dynamics. Our study allows us to identify the dependence of these features on the external parameters, thereby giving us insight into how macroscopic properties arise from microscopic dynamics in this particular nonlinear process. Moreover, we find peculiar spectral characteristics of the squeezing which are unique to this system, and which we trace back to the interplay of the various time scales of the dynamics. In a more general context, our study is connected to previous work on the quantum features of the spectrum of resonance fluorescence [27,28,33-40], and to recent experimental and theoretical studies on quantum correlations in the light scattered by atoms [1-3,5,41-43], by semiconductor microcavities [44], and by macroscopic mirrors $[45,46]$.

This article is organized as follows. In Sec. II the basic coherent dynamics, giving rise to quantum correlations between the Stokes and anti-Stokes components of the spectrum of resonance fluorescence, are briefly reviewed, and the important time scales are introduced. In Sec. III the theoretical model is described in detail and the relevant scattering processes in the system are identified and discussed. In Sec. IV the spectrum of squeezing is evaluated using quantum Langevin equations; for a quick overview of the main results without the full theoretical elaboration, the reader may first skip this part and jump to Sec. IV C where the squeezing characteristics are calculated for a specific, experimentally achievable physical system. Finally, Sec. V presents the conclusions and an outlook.

\section{EPR ENTANGLEMENT OF LIGHT AT THE CAVITY OUTPUT}

In this section we briefly review the coherent dynamics, described previously in Refs. [25,26], which lead to twomode squeezing between the Stokes and anti-Stokes modes in the light scattered by a trapped, laser-driven atom. We thus first ignore incoherent processes and focus on the pulsed dynamics which can be obtained in a suitable parameter regime with a setup similar to the one shown in Fig. 1.

The trapped atom is coupled to an optical cavity of which two modes are resonant with the Stokes and anti-Stokes sidebands, respectively. For short times the laser-induced resonant interaction between the center-of-mass oscillation, de- noted by annihilation and creation operators $b$ and $b^{\dagger}$, and the two cavity modes, represented by operators $a_{j}$ and $a_{j}^{\dagger}$ $(j=1,2)$, is described by the effective Hamiltonian in the interaction picture

$$
W_{\text {eff }}=i \hbar \chi_{1} a_{1}^{\dagger} b^{\dagger}+i \hbar \chi_{2} a_{2}^{\dagger} b+\text { H.c. }
$$

where the scalars $\chi_{j}$ indicate the strength of the coupling. This Hamiltonian generates periodic dynamics, provided that $\left|\chi_{2}\right|>\left|\chi_{1}\right|$, with an angular frequency

$$
\Theta=\sqrt{\left|\chi_{2}\right|^{2}-\left|\chi_{1}\right|^{2}} \text {. }
$$

The time-evolution of the operators, in the Heisenberg representation, is given by [46]

$$
\begin{aligned}
a_{1}(t)= & \frac{\chi_{1}}{\Theta} b^{\dagger}(0) \sin \Theta t+\frac{1}{\Theta^{2}}\left[\left|\chi_{2}\right|^{2}-\left|\chi_{1}\right|^{2} \cos \Theta t\right] a_{1}(0) \\
& -\frac{\chi_{1} \chi_{2}}{\Theta^{2}}[1-\cos \Theta t] a_{2}^{\dagger}(0), \\
a_{2}(t)= & \frac{\chi_{2}}{\Theta} b(0) \sin \Theta t+\frac{\chi_{1} \chi_{2}}{\Theta^{2}}[1-\cos \Theta t] a_{1}^{\dagger}(0)-\frac{1}{\Theta^{2}}\left[\left|\chi_{1}\right|^{2}\right. \\
& \left.-\left|\chi_{2}\right|^{2} \cos \Theta t\right] a_{2}(0), \\
b(t)= & b(0) \cos \Theta t+\frac{1}{\Theta}\left[-\chi_{2}^{*} a_{2}(0)+\chi_{1} a_{1}^{\dagger}(0)\right] \sin \Theta t .
\end{aligned}
$$

In general these solutions describe tripartite entanglement among cavity modes and center-of-mass oscillator [46]. An interesting situation is found after half a period, for $T_{\pi}$ $=\pi / \Theta$. At this time (modulus $2 \pi$ ) the center-of-mass oscillator is uncorrelated with the cavity modes, which exhibit EPR-type entanglement [25,26].

Clearly, this description is approximate, and valid only when incoherent processes can be neglected. In the present work we consider the situation in which the atom is continuously driven by the laser field, such that quantum noise and dissipative processes affect the dynamics relevantly. We show that steady state entanglement, i.e., quantum-correlated spectral fluctuations in the two-mode cavity output field, is found also under these conditions. The details of this entanglement will depend on the comparison between the time scale set by the coherent dynamics $\Theta^{-1}$ and the time scales of the dissipative processes $\kappa^{-1}$ for loss of photons from the cavity and $\gamma^{-1}$ for spontaneous scattering from the atom. In particular, we will show that the squeezing spectrum shows distinct, qualitatively different features in the regimes $\Theta$ $<\kappa, \Theta=\kappa$, and $\Theta>\kappa$. The reader is referred to Sec. IV C, where the spectra for different parameter regimes are reported.

\section{SCATTERING PROCESSES}

The purpose of this section is to discuss the coherent and incoherent scattering processes determining the dynamics of the system. We will present these processes using physical pictures derived from the scattering matrix under moderate simplifications, in order to illustrate the more rigorous deri- 
vations presented in the subsequent section. We first introduce the model, and then identify the scattering processes and determine the corresponding rates.

\section{A. Model}

We consider an atom of mass $M$ inside an optical resonator and driven by a laser. The atomic motion is confined by an external potential, which we assume sufficiently steep in the radial direction so that the motion in this plane can be considered frozen out. We denote by $x$ the axis of the remaining one-dimensional atomic center-of-mass motion. Moreover, we assume that only the atomic dipole transition between ground state $|g\rangle$ and excited state $|e\rangle$ couples relevantly to the fields, such that we can restrict the electronic dynamics to these two states. The atomic dipole is laser driven, and it couples to two modes $(j=1,2)$ of the resonator, as well as to the external modes of the electromagnetic field. The cavity modes couple also to the external modes of the electromagnetic field through the imperfect mirrors of the resonator. The total dynamics is governed by the Hamiltonian

$$
H=H_{0}+W,
$$

where $H_{0}$ is the self-energy of the system of atom and fields and $W$ describes their mutual interaction, as well as the coupling between the cavity modes and the external modes through the finite transmission at the cavity mirrors. We now introduce each term in detail, and discuss the dynamics in the reference frame of the laser at the angular frequency $\omega_{L}$. We decompose $H_{0}$ according to

$$
H_{0}=H_{a}+H_{c}+H_{\mathrm{EMF}} .
$$

Here, $H_{a}$ is the Hamiltonian for the relevant atomic degrees of freedom

$$
H_{a}=-\hbar \Delta|e\rangle\langle e|+H_{\mathrm{mec}},
$$

where $\Delta=\omega_{L}-\omega_{0}$ is the detuning of the laser from the dipole transition at the angular frequency $\omega_{0}$, and

$$
H_{\text {mec }}=\hbar \nu\left(b^{\dagger} b+\frac{1}{2}\right)
$$

describes the harmonic motion of the atomic center of mass at angular frequency $\nu$, as determined by an external potential, where $b, b^{\dagger}$ are the annihilation and creation operators, respectively, of a quantum of vibrational energy $\hbar \nu$. In particular, the atomic position is given by $x=\sqrt{\hbar / 2 M \nu}\left(b+b^{\dagger}\right)$. We denote by $|n\rangle$ the eigenstates of $H_{\mathrm{mec}}$ at energy $\hbar \nu(n+1 / 2)$. The Hamiltonian for the cavity modes, which couple appreciably to the dipole transition, is

$$
H_{c}=-\sum_{j=1,2} \hbar \delta_{j} a_{j}^{\dagger} a_{j},
$$

where $\delta_{j}=\omega_{L}-\omega_{j}$ are the detunings of the laser from the frequencies $\omega_{j}$ of two optical modes, and $a_{j}, a_{j}^{\dagger}$ are the respective annihilation and creation operators of a quantum of energy $\hbar \omega_{j}$, i.e., a photon in mode $j$. We denote by $\left|n_{1}, n_{2}\right\rangle$ the eigenstates of $H_{c}$ at energy $-\hbar \delta_{1} n_{1}-\hbar \delta_{2} n_{2}$, and consider the situation in which the mode frequencies fulfill the relation

$$
\omega_{2}-\omega_{1}=2 \nu^{\prime},
$$

where

$$
\nu^{\prime}=\nu+\delta \nu
$$

and $\delta \nu$ takes into account radiative shifts, such that cavity modes 1 and 2 can be simultaneously resonant with the Stokes and the anti-Stokes transitions. This contribution will be discussed in Sec. III B 4 and determined in Sec. IV A.

Finally, the modes of the electromagnetic field external to the cavity possess the free Hamiltonian

$$
H_{\mathrm{EMF}}=-\hbar \sum_{\mathbf{k}_{j}} \delta_{\mathbf{k}_{j}} r_{\mathbf{k}_{j}}^{\dagger} r_{\mathbf{k}_{j}}-\hbar \sum_{\mathbf{k}_{s}} \delta_{\mathbf{k}_{s}} r_{\mathbf{k}_{s}}^{\dagger} r_{\mathbf{k}_{s}},
$$

where $r_{\lambda}, r_{\lambda}^{\dagger}$ are annihilation and creation operators, respectively, of a photon at angular frequency $\omega_{\lambda}=\omega_{L}-\delta_{\lambda}$, wavevector $\mathbf{k}_{\lambda}$, and polarization $\mathbf{e}_{\lambda}$. Here, the subscripts $\lambda$ $=\mathbf{k}_{s}$ and $\lambda=\mathbf{k}_{j}$ indicate the modes of the field which couple to the dipole and to the cavity modes (through the mirrors), respectively. The interaction term

$$
W=H_{a L}+H_{a c}+W_{\mathbf{k}_{s}}+W_{\mathbf{k}_{j}}
$$

describes the couplings among atom and fields, decomposed into four terms which correspond to the coupling between atom and laser $\left(H_{a L}\right)$, atom and cavity modes $\left(H_{a c}\right)$, atom and modes of the external electromagnetic field $\left(W_{\mathbf{k}_{s}}\right)$, and cavity modes and external electromagnetic field $\left(W_{\mathbf{k}_{j}}^{s}\right)$. We discuss these terms in the Lamb-Dicke regime, when the atomic motion is well localized over the wavelengths of the fields, such that the Lamb-Dicke parameter $\eta=\sqrt{\hbar k^{2} / 2 M \nu}$ is small, $\eta \ll 1$. At lowest order in $\eta$, the coupling between laser and dipole has the form [47]

$$
\begin{aligned}
H_{a L}= & \hbar \Omega \sigma^{\dagger}\left[\left(1-\frac{\eta^{2}}{2} \cos ^{2} \theta_{L}\left(2 b^{\dagger} b+1\right)\right)\right. \\
& \left.+i \eta \cos \theta_{L}\left(b^{\dagger}+b\right)+O\left(\eta^{2}\right)\right]+ \text { H.c. },
\end{aligned}
$$

with $\sigma=|g\rangle\langle e|$ the dipole lowering operator and $\sigma^{\dagger}$ its adjoint, $\Omega$ the Rabi frequency, and $\theta_{L}$ the angle between the direction of propagation of the laser and the motional axis $\hat{x}$. In what follows we denote the moduli of all relevant wave vectors by $k$, as their differences are negligible. The coupling between the dipole and the cavity modes is represented by

$$
\begin{aligned}
H_{a c}= & \hbar \sum_{j=1,2} g_{j} \cos \phi_{j} a_{j} \sigma^{\dagger}\left[\left(1-\frac{\eta^{2}}{2} \cos ^{2} \theta_{c}\left(2 b^{\dagger} b+1\right)\right)\right. \\
& \left.-\eta \cos \theta_{c} \tan \phi_{j}\left(b^{\dagger}+b\right)\right]+ \text { H.c. }+O\left(\eta^{2}\right),
\end{aligned}
$$

where $g_{j}$ is the coupling strength of the dipole to mode $j$, and the cavity axis forms an angle $\theta_{c}$ with the axis $\hat{x}$ of the motion. The angle $\phi_{j}$ takes into account the position of the trap center inside the standing wave of the cavity. Finally, the terms 


$$
\begin{gathered}
W_{\mathbf{k}_{s}}=\sum_{\mathbf{k}_{s}} \hbar g_{\mathbf{k}_{s}} \sigma^{\dagger} r_{\mathbf{k}_{s}}\left[\left(1-\frac{\eta^{2}}{2} \cos ^{2} \theta_{\mathbf{k}_{s}}\left(2 b^{\dagger} b+1\right)\right)\right. \\
\left.+i \eta \cos \theta_{\mathbf{k}_{s}}\left(b+b^{\dagger}\right)+O\left(\eta^{2}\right)\right]+ \text { H.c. } \\
W_{\mathbf{k}_{j}}=\sum_{\mathbf{k}_{j}} \hbar g_{\mathbf{k}_{j}}\left(a_{j}^{\dagger} r_{\mathbf{k}_{j}}+\text { H.c. }\right)
\end{gathered}
$$

describe the coupling of atom and cavity to the modes of the external electromagnetic (EM) field. Here, $W_{\mathbf{k}_{s}}$ is the coupling of the dipole, at Rabi frequencies $g_{\mathbf{k}_{s}}$, with the external modes, whose wave vectors form angles $\theta_{\mathbf{k}_{s}}$ with the motional axis. This coupling gives rise to the finite linewidth $\gamma$ of the excited state $\gamma=2 \pi \rho_{\mathbf{k}_{s}}\left(\omega_{0}\right)\left|g_{\mathbf{k}_{s}}\left(\omega_{0}\right)\right|^{2}$, with $\rho_{\mathbf{k}_{s}}\left(\omega_{0}\right)$ density of states of the EM-field coupling to the atomic dipole at angular frequency $\omega_{0}$. The term $W_{\mathbf{k}_{j}}$ describes the coupling of the cavity modes with the external modes at strength $g_{\mathbf{k}_{j}}$. This coupling gives rise to the linewidth of the cavity modes $\kappa_{j}=\pi\left|g_{\mathbf{k}_{j}}\right|^{2} \rho_{\mathbf{k}_{j}}\left(\omega_{j}\right)$, with $\rho_{\mathbf{k}_{j}}\left(\omega_{j}\right)$ density of states of the EMfield coupling to the cavity modes at angular frequency $\omega_{j}$.

\section{B. Basic scattering processes}

We consider the limit in which the atom is far-detuned from cavity modes and laser, $|\Delta| \gg \gamma, \delta_{j}, g_{j}, \Omega$. In this limit all terms of $W$ are weak perturbations to the dynamics. We assume that the system is in the initial state

$$
\left|\psi_{i}\right\rangle=\left|g, n ; 0_{1}, 0_{2} ; 0_{\mathbf{k}_{j}} ; 0_{\mathbf{k}_{s}}\right\rangle,
$$

with energy $E_{i}=\hbar \nu n$, where the atom is in the ground state $|g\rangle$, the center-of-mass oscillator is in the number state $|n\rangle$, and the cavity modes and the external EM-field are in the vacuum state $\left|0_{1}, 0_{2} ; 0_{\mathbf{k}_{j}} ; 0_{\mathbf{k}_{s}}\right\rangle$. The scattering matrix elements between the initial state and all possible final states $\left|\psi_{f}\right\rangle$, with energy $E_{f}$, have the form

$$
\mathcal{S}_{i f}=\delta_{i f}-2 \pi i \delta\left(E_{f}-E_{i}\right) \mathcal{T}_{i f},
$$

where $\delta_{i f}$ is the Kronecker delta, $\delta\left(E_{f}-E_{i}\right)$ is a delta function giving energy conservation between initial and final states, and $\mathcal{T}_{\text {if }}$ is the transition matrix to be evaluated in lowest order in perturbation theory

$$
\mathcal{T}_{i f}=\left\langle\psi_{f}|W| \psi_{i}\right\rangle+\left\langle\psi_{f}\left|W \frac{1}{E_{i}-H_{\text {eff }}} W\right| \psi_{i}\right\rangle,
$$

with

$$
H_{\mathrm{eff}}=-\hbar\left(\Delta+i \frac{\gamma}{2}\right)|e\rangle\langle e|+\hbar \nu b^{\dagger} b-\hbar \sum_{j=1,2}\left(\delta_{j}+i \kappa_{j}\right) a_{j}^{\dagger} a_{j}
$$

We now consider all possible scattering transitions to resonant states, i.e., to final states $\left|\psi_{f}\right\rangle$ at energy $E_{f}=E_{i}$.

\section{Scattering of laser photons into the external EM field}

We consider the scattering of a laser photon into the external EM field by spontaneous emission, hence coupling of
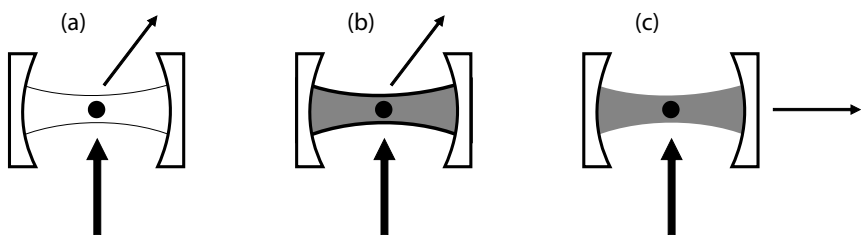

FIG. 2. Basic scattering processes: (a) A laser photon is absorbed and emitted by the atom, without coupling to the cavity mode; (b) a laser photon is scattered into the cavity mode, and then rescattered by the atom into the external modes of the electromagnetic field; and (c) a laser photon is scattered by the atom into the cavity mode, and then transmitted by the cavity mirror into the modes of the external electromagnetic field.

$\left|\psi_{i}\right\rangle$ to the final states $\left|\psi_{\mathbf{k}_{s}}\right\rangle=\left|g, n^{\prime} ; 0_{1}, 0_{2} ; 0_{\mathbf{k}_{j}} ; 1_{\mathbf{k}_{s}}\right\rangle$. This process is sketched in Fig. 2(a). Here, the coupling with the cavity mode is neglected, as the cavity is far-detuned from the dipole, and the rate of this process can be approximated by the scattering rate of the atom in free space

$$
\Gamma_{i f}^{\mathrm{sp}} \approx \frac{1}{\gamma}\left(\left|t_{0}^{\mathrm{sp}}\right|^{2} \delta_{n^{\prime}, n}+\left|t_{+1}^{\mathrm{sp}}\right|^{2}(n+1) \delta_{n^{\prime}, n+1}+\left|t_{-1}^{\mathrm{sp}}\right|^{2} n \delta_{n^{\prime}, n-1}\right)
$$

where

$$
\begin{gathered}
t_{0}^{\mathrm{sp}}=\frac{\gamma \Omega}{\Delta+i \gamma / 2}, \\
t_{+1}^{\mathrm{sp}}=\eta \gamma \Omega\left(\frac{\cos \theta_{L}}{\Delta-\nu+i \gamma / 2}+\frac{\cos \theta_{\mathbf{k}_{\mathrm{s}}}}{\Delta+i \gamma / 2}\right), \\
t_{-1}^{\mathrm{sp}}=\eta \gamma \Omega\left(\frac{\cos \theta_{L}}{\Delta+\nu+i \gamma / 2}+\frac{\cos \theta_{\mathbf{k}_{\mathrm{s}}}}{\Delta+i \gamma / 2}\right) .
\end{gathered}
$$

The process described by amplitude (19) does not affect the dynamics of either the cavity modes or the center-of-mass motion. In contrast, amplitudes (20) and (21) are coherent superpositions of scattering processes involving, respectively, the mechanical effect of the laser and the emitted photon on the atomic motion $[28,30]$, thereby affecting the coherence of the motional state. Their rate is $\gamma_{b} \approx \eta^{2}\left(\cos ^{2} \theta_{L}\right.$ $+\alpha) \gamma \Omega^{2} / \Delta^{2}$, where $\alpha$ describes the angular dispersion of the spontaneously emitted photons, determined by the quantum numbers of the atomic transition [48].

\section{Scattering of laser photons into the cavity modes}

Next, we discuss the process where a laser photon is scattered into one of the cavity modes, thereby coupling the initial state $\left|\psi_{i}\right\rangle$ to the states $\left|\psi_{1}\right\rangle=\left|g, n^{\prime} ; 1_{1}, 0_{2} ; 0_{\boldsymbol{k}_{j}} ; 0_{\boldsymbol{k}_{\boldsymbol{s}}}\right\rangle$ or $\left|\psi_{2}\right\rangle$ $=\left|g, n^{\prime} ; 0_{1}, 1_{2} ; 0_{k_{j}} ; 0_{\boldsymbol{k}_{\boldsymbol{s}}}\right\rangle$. As these states are not stable, but resonantly coupled to the continuum of states $\left|g, n^{\prime} ; 0_{1}, 0_{2} ; 1_{k_{j}} ; 0_{\boldsymbol{k}_{\boldsymbol{s}}}\right\rangle$ by cavity decay, the correct final states of these scattering processes describe the processes sketched in Fig. 2(c) and have the form 


$$
\left|\psi_{\boldsymbol{k}_{j}}\right\rangle=\sqrt{Z_{\boldsymbol{k}_{j}}}\left[1+\frac{Q_{j}}{\left(E_{\boldsymbol{k}_{j}}-H\right)} W_{\boldsymbol{k}_{j}}\right]\left|\psi_{j}\right\rangle,
$$

where $Q_{j}$ projects onto the subspace orthogonal to $\left|\psi_{j}\right\rangle$, and $Z_{\boldsymbol{k}_{j}}$ ensures the normalization of the state. Furthermore, $Z_{\boldsymbol{k}_{j}}$ gives the occupation probability of state $\left|\psi_{j}\right\rangle$, since $Z_{k_{j}}=\left|\left\langle\psi_{j} \mid \psi_{k_{j}}\right\rangle\right|^{2}$.

The coupling rate between state $\left|\psi_{i}\right\rangle$ and states $\left|\psi_{\boldsymbol{k}_{j}}\right\rangle$ takes the form

$$
\begin{aligned}
\Gamma_{i f_{j}}^{\mathrm{cav}} \approx & \frac{2 \kappa_{j}}{\delta_{j}^{2}+\kappa_{j}^{2}}\left|t_{0}^{\mathrm{cav}}\right|^{2} \delta_{n^{\prime}, n}+\frac{2 \kappa_{j}}{\left(\delta_{j}-\nu\right)^{2}+\kappa_{j}^{2}}\left|t_{j,+}^{\mathrm{cav}}\right|^{2}(n+1) \delta_{n^{\prime}, n+1} \\
& +\frac{2 \kappa_{j}}{\left(\delta_{j}+\nu\right)^{2}+\kappa_{j}^{2}}\left|t_{j,-}^{\mathrm{cav}}\right|^{2} n \delta_{n^{\prime}, n-1}
\end{aligned}
$$

where

$$
\begin{gathered}
t_{0}^{\mathrm{cav}}=\Omega g_{j}^{*} \cos \phi_{j} \frac{1}{\Delta+i \gamma / 2}, \\
t_{j,+}^{\mathrm{cav}}=\eta \Omega g_{j}^{*} \cos \phi_{j}\left[\frac{i \cos \theta_{L}}{\Delta-\nu+i \gamma / 2}-\frac{\cos \theta_{c} \tan \phi_{j}}{\Delta+i \gamma / 2}\right], \\
t_{j,-}^{\mathrm{cav}}=\eta \Omega g_{j}^{*} \cos \phi_{j}\left[\frac{i \cos \theta_{L}}{\Delta+\nu+i \gamma / 2}-\frac{\cos \theta_{c} \tan \phi_{j}}{\Delta+i \gamma / 2}\right] .
\end{gathered}
$$

As in Eqs. (20) and (21), we recognize on the right-hand side of Eqs. (25) and (26) the coherent addition of two scattering amplitudes, here representing the mechanical effects of the laser and of the cavity, respectively $[49,50]$. These processes are at the basis of the coherent coupling between the atomic motion and the cavity modes described by Hamiltonian (1), where $\chi_{1}=-i t_{1,+}^{\mathrm{cav}}$ and $\chi_{2}=-i t_{2,-}^{\mathrm{cav}}$. We are interested in the regime where energy can be stored in the cavity modes through this coupling, which requires $\left|\chi_{1}\right|,\left|\chi_{2}\right| \gg \gamma_{b}$ as a necessary condition. In this situation, it is visible from the equations that in the limit $\kappa_{j} \ll \nu$, by choosing $\delta_{1}=\nu$ and $\delta_{2}=-\nu$ one can achieve the optimum enhancement of the scattering of a laser photon into mode 1 accompanied by the excitation of the motion by one vibrational quantum, and of the scattering of a laser photon into mode 2 accompanied by the deexcitation of the motion by one vibrational quantum. Note that these scattering terms $t_{j, \pm}^{\mathrm{cav}}$ have an incoherent component which scales with $\gamma /|\Delta \pm \nu|$. Therefore, in general coherent dynamics can only be achieved when $\gamma \ll|\Delta|$, on a time scale such that incoherent terms are negligible. Moreover, the condition $\gamma$ $\ll \nu$ is also required in order to create quantum correlations between the two cavity modes, since the difference between the two coupling strengths $\chi_{1}$ and $\chi_{2}$ determines the typical time scale on which entanglement is established, see Sec. II and Ref. [26].

\section{Scattering of cavity photons into the external EM field}

Assuming that photons have been coherently scattered into the cavity modes, they can be reabsorbed by the atom and emitted spontaneously into the external EM field, as sketched in Fig. 2(b). In order to focus on the evaluation of the corresponding element of the scattering matrix, we con- sider the regime of very small cavity loss rate, i.e., we assume stable cavity modes and ignore, for the clarity of the picture, cavity decay. Be the initial state

$$
\left|\psi_{i, m}\right\rangle=\left|g, n ; m_{1}, m_{2} ; 0_{\mathbf{k}_{j}} ; 0_{\mathbf{k}_{s}}\right\rangle
$$

at energy $E_{i, m}=\hbar \nu n-\hbar m_{1} \delta_{1}-\hbar m_{2} \delta_{2}$, with the atom in $|g\rangle$, the center-of-mass oscillator in the number state $|n\rangle$, the cavity modes in the Fock states $\left|m_{1}\right\rangle$ and $\left|m_{2}\right\rangle$, and the external EM field in the vacuum state, $\left|0_{\mathbf{k}_{j}} ; 0_{\mathbf{k}_{s}}\right\rangle$. This state is coupled to the states

$$
\left|\psi_{f, m_{1}^{\prime}}\right\rangle=\left|g, n ; m_{1}-1, m_{2} ; 0_{\mathbf{k}_{j}} ; 1_{\mathbf{k}_{s}}\right\rangle
$$

$$
\left|\psi_{f, m_{2}^{\prime}}\right\rangle=\left|g, n ; m_{1}, m_{2}-1 ; 0_{\mathbf{k}_{j}} ; 1_{\mathbf{k}_{s}}\right\rangle
$$

by absorption of a cavity photon and spontaneous emission. We evaluate the corresponding rate under the assumption, that $\tan \phi_{j}=0$, i.e., there are no mechanical effects of the resonator on the atom at first order in $\eta$, and find an effective loss rate of the cavity modes

$$
\Gamma_{i f_{j}}^{\mathrm{cav}-\mathrm{sp}}=\gamma\left|g_{j}\right|^{2}\left|\frac{\sqrt{m_{j}}}{\Delta-\delta_{j}+i \gamma / 2}\right|^{2} .
$$

It should be noted that these processes arise from atomic scattering of a laser photon into the cavity modes, which is then rescattered by atomic emission into the external modes of the EM field. Hence, these processes can interfere with atomic scattering of a laser photon, in the limit discussed in Sec. III B 1, in which the coupling to the cavity plays no role. In these calculations we have not considered the coherent addition of these two noise effects, but we will consider phase relations and possible interference in these noise sources when studying the dynamics with the quantum Langevin equations in Sec. IV A.

\section{4. ac Stark shift of the ground state energy}

Since the efficiency of production of two-mode squeezed light is based on the resonant enhancement of two-photon processes, it is important to consider systematically radiative corrections to the resonance frequencies in the implementation of the dynamics in Sec. II. Therefore, we now evaluate corrections to the energy of state $\left|\psi_{i, m}\right\rangle$, Eq. (27), due to far-off resonance coupling in the limit of very small cavity decay rates. When considering the ac-Stark shift of state $\left|\psi_{i, m}\right\rangle$, we find three contributions, each associated to different kinds of coupling. (i) The ac-Stark shift due to the off-resonant laser coupling with the excited state at zero order in the mechanical effects, $\delta \omega_{0} \sim \Omega^{2} / \Delta$ for $|\Delta| \gg \gamma$. It leads to a shift $\delta \omega_{0}$ of the dipole resonance frequency. The mechanical effects of the laser on the atoms give rise to (ii) a contribution which is linear in the number of vibrational ex- 
citation, and can hence be considered a renormalization of the trap frequency. This ac-Stark shift reads

$$
\begin{aligned}
\delta \nu_{b} \approx & \eta^{2} \cos ^{2} \theta_{L} \Omega^{2} b^{\dagger} b \\
& \times \operatorname{Re}\left\{\frac{1}{\Delta+\nu+i \gamma / 2}+\frac{1}{\Delta-\nu+i \gamma / 2}-\frac{1}{\Delta+i \gamma / 2}\right\} \\
= & \eta^{2} \cos ^{2} \theta_{L} \Omega^{2} b^{\dagger} b \\
& \times\left(\frac{2 \Delta\left(\Delta^{2}-\nu^{2}+\gamma^{2} / 4\right)}{\left(\Delta^{2}-\nu^{2}+\gamma^{2} / 4\right)^{2}+\gamma^{2} \nu^{2}}-\frac{\Delta}{\Delta^{2}+\gamma^{2} / 4}\right) .
\end{aligned}
$$

Finally, off-resonant coupling of the cavity mode with the dipole transition gives rise to an ac-Stark shift of the cavity mode levels, which reads at leading order

$$
\delta \omega_{j} \approx \frac{\left|g_{j}\right|^{2} \cos ^{2} \phi_{j}\left(\Delta-\delta_{j}\right)}{\left(\Delta-\delta_{j}\right)^{2}+\gamma^{2} / 4} a_{j}^{\dagger} a_{j}+O\left(\eta^{2}\right) .
$$

These shifts are in general not small and should be taken into account, when aiming at the resonant enhancement of certain processes over others. It should be remarked that the correction to $\delta \omega_{j}$ in Eq. (32) which is at second order in $\eta$ arises from the mechanical effects of the interaction between resonator and center-of-mass motion. This term is nonlinear, as it is a shift which depends on the number of vibrational excitation, but is a negligible contribution to $\delta \omega_{j}$. On the other hand, this term gives rise to an additional contribution to the ac-Stark shift of the center-of-mass motion, which is of the same order as $\delta \nu_{b}$ and depends on the number of photons. Its effect is detrimental, as the resulting spectrum of the centerof-mass excitations deviates from the one of a harmonic oscillator. In the system we consider we will neglect this contribution, focussing onto the regime in which the mechanical effects of the cavity mode can be neglected. This corresponds to situations, where the motion, for instance, is almost orthogonal to the cavity wave vector $\left|\cos \theta_{c}\right| \ll 1$.

\section{SPECTRUM OF LIGHT AT THE CAVITY OUTPUT}

In this section we evaluate the spectrum of the light transmitted by the cavity mirror. The spectrum is best evaluated using the quantum Langevin equations for the operators $a_{j}$, $a_{j}^{\dagger}$, and $b$. The equations we obtain are rather involved, however, the physical meaning of each term can be identified by comparison with the rates of the scattering processes discussed in the previous section.

\section{A. Quantum Langevin equations}

We shall study the dynamics using the quantum Langevin equations (QLE) of the system. For convenience, we write the interaction Hamiltonian of the atom with the laser and the cavity fields as

$$
H_{\mathrm{int}}=H_{a L}+H_{a c}=\hbar\left(\sigma^{\dagger} B+\sigma B^{\dagger}\right),
$$

where

$$
\begin{aligned}
B= & \Omega\left(1-\frac{\eta^{2}}{2} \cos ^{2} \theta_{L}\left(2 b^{\dagger} b+1\right)\right)+i \eta \Omega \cos \theta_{L}\left(b^{\dagger}+b\right) \\
& +\sum_{j=1,2} g_{j} \cos \phi_{j} a_{j}\left(1-\frac{\eta^{2}}{2} \cos ^{2} \theta_{c}\left(2 b^{\dagger} b+1\right)\right) \\
& -\eta \cos \theta_{c} g_{j} \sin \phi_{j} a_{j}\left(b^{\dagger}+b\right) .
\end{aligned}
$$

The QLE read

$$
\dot{a}_{1}(t)=i \delta_{1} a_{1}(t)+i \sigma(t)\left[B(t)^{\dagger}, a_{1}(t)\right]-\kappa_{1} a_{1}(t)+\sqrt{2 \kappa_{1}} a_{1}^{\text {in }}(t),
$$

$$
\dot{a}_{2}(t)=i \delta_{2} a_{1}(t)+i \sigma(t)\left[B(t)^{\dagger}, a_{2}(t)\right]-\kappa_{2} a_{2}(t)+\sqrt{2 \kappa_{2}} a_{2}^{\text {in }}(t),
$$

$$
\begin{aligned}
\dot{b}(t)= & -i \nu b(t)+i \sigma(t)\left[B(t)^{\dagger}, b(t)\right]+i \sigma(t)^{\dagger}[B(t), b(t)] \\
& -\kappa_{b} b(t)+\sqrt{2 \kappa_{b}} b^{\mathrm{in}}(t), \\
\dot{\sigma}(t)= & {\left[i \Delta-\frac{\gamma}{2}\right] \sigma(t)+\sigma_{z}(t)\left[i B(t)+\sqrt{\gamma} f^{\mathrm{in}}(t)\right], } \\
\dot{\sigma}_{z}(t)= & 2 i \sigma(t) B(t)^{\dagger}-2 i \sigma^{\dagger}(t) B(t)-\gamma\left[\sigma_{z}(t)+1\right] \\
& -2 \sigma^{\dagger}(t) \sqrt{\gamma} f^{\mathrm{in}}(t)-2 \sigma(t) \sqrt{\gamma} f^{\mathrm{in}}(t)^{\dagger},
\end{aligned}
$$

where $\sigma_{z}=\sigma^{\dagger} \sigma-\sigma \sigma^{\dagger}$, and we have introduced the vacuum input noises $a_{j}^{\text {in }}(t)(j=1,2)$ of the cavity modes with corresponding decay rate $\kappa_{j}$, the spontaneous emission noise $f^{\text {in }}(t)$ at rate $\gamma$, and we also added a phenomenological input noise $b^{\text {in }}(t)$ acting on the atom's motion, describing the heating at rate $\kappa_{b}$ due to the fluctuations of the trap potential. These four noise sources are mutually uncorrelated and have zero mean, while their second-order correlations have the form

$$
\begin{gathered}
\left\langle a_{1}^{\mathrm{in}}(t) a_{1}^{\mathrm{in}}\left(t^{\prime}\right)^{\dagger}\right\rangle=\left\langle a_{2}^{\mathrm{in}}(t) a_{2}^{\mathrm{in}}\left(t^{\prime}\right)^{\dagger}\right\rangle=\delta\left(t-t^{\prime}\right), \\
\left\langle f^{\mathrm{in}}(t) f^{\mathrm{in}}\left(t^{\prime}\right)^{\dagger}\right\rangle=\delta\left(t-t^{\prime}\right), \\
\left\langle b^{\mathrm{in}}(t) b^{\mathrm{in}}\left(t^{\prime}\right)^{\dagger}\right\rangle=(\bar{N}+1) \delta\left(t-t^{\prime}\right), \\
\left\langle b^{\mathrm{in}}(t)^{\dagger} b^{\mathrm{in}}\left(t^{\prime}\right)\right\rangle=\bar{N} \delta\left(t-t^{\prime}\right),
\end{gathered}
$$

where $\bar{N}$ is mean thermal vibrational number of the effective thermal reservoir coupling to the atom center-of-mass motion [51].

We assume that the laser is red-detuned and far-off resonance from the atomic transition, i.e., $\Delta$ is negative and $|\Delta|$ is much larger than all the other parameters. This allows us to eliminate adiabatically the atomic internal degrees of freedom, and to assume that the atom always remains in the ground state $|g\rangle$, that is, $\sigma_{z}(t) \approx-1$. Therefore we neglect the time evolution of $\sigma_{z}$, Eq. (39), while Eq. (38) becomes

$$
\dot{\sigma}(t)=-(\gamma / 2-i \Delta) \sigma(t)-i B(t)-\sqrt{\gamma} f^{\mathrm{in}}(t),
$$

whose formal solution is 


$$
\begin{aligned}
\sigma(t)= & e^{-(\gamma / 2-i \Delta) t} \sigma(0)-\int_{0}^{t} \mathrm{~d} s \mathrm{e}^{-(\gamma / 2-i \Delta) s}[i B(t-s) \\
& \left.+\sqrt{\gamma} f^{\mathrm{in}}(t-s)\right] .
\end{aligned}
$$

We now insert solution (45) into the other QLE and neglect the transient term because we are interested in the dynamics at times which are much larger than $1 /|\Delta|$. We obtain

$$
\begin{gathered}
\dot{a}_{1}(t)=i \delta_{1} a_{1}(t)+\int_{0}^{t} \mathrm{~d} s \mathrm{e}^{-(\gamma / 2-i \Delta) s}\left[B(t-s)-i \sqrt{\gamma} f^{\mathrm{in}}(t-s)\right]\left[B(t)^{\dagger}, a_{1}(t)\right]-\kappa_{1} a_{1}(t)+\sqrt{2 \kappa_{1}} a_{1}^{\mathrm{in}}(t), \\
\dot{a}_{2}(t)=i \delta_{2} a_{1}(t)+\int_{0}^{t} \mathrm{~d} s \mathrm{e}^{-(\gamma / 2-i \Delta) s}\left[B(t-s)-i \sqrt{\gamma} f^{\mathrm{in}}(t-s)\right]\left[B(t)^{\dagger}, a_{2}(t)\right]-\kappa_{2} a_{2}(t)+\sqrt{2 \kappa_{2}} a_{2}^{\mathrm{in}}(t), \\
\dot{b}(t)=-i \nu b(t)+\int_{0}^{t} \mathrm{~d} s \mathrm{e}^{-(\gamma / 2-i \Delta) s}\left[B(t-s)-i \sqrt{\gamma} f^{\mathrm{in}}(t-s)\right]\left[B(t)^{\dagger}, b(t)\right]-\int_{0}^{t} \mathrm{~d} s \mathrm{e}^{-(\gamma / 2+i \Delta) s}\left[B(t-s)^{\dagger}+i \sqrt{\gamma} f^{\mathrm{in}}(t-s)^{\dagger}\right][B(t), b(t)] \\
-\kappa_{b} b(t)+\sqrt{2 \kappa_{b}} b^{\mathrm{in}}(t),
\end{gathered}
$$

where we have not taken care of operator ordering, since, as we shall see, within the validity limit of our treatment these integral terms will generate only linear contributions. that

At this point, we choose the laser angular frequency $\omega_{L}$ so

$$
\delta_{1}=\nu^{\prime} ; \quad \delta_{2}=-\nu^{\prime},
$$

namely, the laser frequency is tuned symmetrically between the mode frequencies, which are spaced by a quantity $2 \nu^{\prime}$. The angular frequency $\nu^{\prime} \simeq \nu$, and takes into account the ac-Stark shifts due to the mechanical coupling with laser and cavity modes, see Sec. III B 4, so that the two cavity modes are resonant with the motional sidebands of the laser light. Together with this choice of the laser frequency, we assume that the motional sidebands are well resolved, that is, $\nu$ $\gg\left|g_{j}\right|, \Omega, \kappa_{j}$.

In order to identify the resonant process, we move to a frame rotating at the effective vibrational angular frequency $\nu^{\prime} \simeq \nu$, (which has to be determined by solving the QLE) and we will neglect in the QLE all the terms oscillating at $\nu^{\prime}$ or larger. Denoting the slowly varying quantities by $\widetilde{a}_{1}^{\dagger}(t)$ $\equiv e^{i \nu^{\prime} t} a_{1}^{\dagger}(t), \widetilde{a}_{2}(t) \equiv e^{i \nu^{\prime} t} a_{2}(t), \widetilde{b}(t) \equiv e^{i \nu^{\prime} t} b(t)$, after explicitly evaluating the commutators we obtain

$$
\begin{aligned}
& \dot{\tilde{a}}_{1}(t)=i\left(\nu^{\prime}-\delta_{1}\right) \tilde{a}_{1}^{\dagger}(t)+\int_{0}^{t} \mathrm{~d} s \mathrm{e}^{-(\gamma / 2+i \Delta) s}\left[B(t-s)^{\dagger} e^{i \nu^{\prime} t}+i \sqrt{\gamma} f^{\mathrm{in}}(t-s)^{\dagger} e^{i \nu^{\prime} t}\right]\left[-g_{1} \cos \phi_{1}\left(1-\frac{\eta^{2}}{2} \cos ^{2} \theta_{c}\left(2 \tilde{b}^{\dagger} \tilde{b}+1\right)\right)\right. \\
& \left.+\eta g_{1} \sin \phi_{1} \cos \theta_{c}\left[\tilde{b}(t) e^{-i \nu^{\prime} t}+\widetilde{b}^{\dagger}(t) e^{i \nu^{\prime} t}\right]\right]-\kappa_{1} \widetilde{a}_{1}^{\dagger}(t)+\sqrt{2 \kappa_{1}} \tilde{a}_{1}^{\text {in }}(t)^{\dagger}, \\
& \dot{\tilde{a}}_{2}(t)=i\left(\nu^{\prime}+\delta_{2}\right) \widetilde{a}_{2}(t)+\int_{0}^{t} \mathrm{~d} s \mathrm{e}^{-(\gamma / 2-i \Delta) s}\left[B(t-s) e^{i \nu^{\prime} t}-i \sqrt{\gamma} f^{\mathrm{in}}(t-s) e^{i \nu^{\prime} t}\right]\left[-g_{2}^{*} \cos \phi_{2}\left(1-\frac{\eta^{2}}{2} \cos ^{2} \theta_{c}\left(2 \tilde{b}^{\dagger} \tilde{b}+1\right)\right)\right. \\
& \left.+\eta g_{2}^{*} \sin \phi_{2} \cos \theta_{c}\left[\widetilde{b}(t) e^{-i \nu^{\prime} t}+\tilde{b}^{\dagger}(t) e^{i \nu^{\prime} t}\right]\right]-\kappa_{2} \widetilde{a}_{2}(t)+\sqrt{2 \kappa_{2}} \widetilde{a}_{2}^{\mathrm{in}}(t) \\
& \dot{\tilde{b}}(t)=i\left(\nu^{\prime}-\nu\right) \tilde{b}(t)+\int_{0}^{t} \mathrm{~d} s \mathrm{e}^{-(\gamma / 2-i \Delta) s}\left[B(t-s) e^{i \nu^{\prime} t}-i \sqrt{\gamma} f^{\mathrm{in}}(t-s) e^{i \nu^{\prime} t}\right]
\end{aligned}
$$




$$
\begin{gathered}
\times\left[i \eta \Omega^{*} \cos \theta_{L}+\eta g_{1}^{*} \sin \phi_{1} \cos \theta_{c} \widetilde{a}_{1}^{\dagger}(t) e^{-i \nu^{\prime} t}+\eta g_{2}^{*} \sin \phi_{2} \cos \theta_{c} \widetilde{a}_{2}^{\dagger}(t) e^{i \nu^{\prime} t}\right] \\
-\int_{0}^{t} \mathrm{~d} s \mathrm{e}^{-(\gamma / 2+i \Delta) s}\left[B(t-s)^{\dagger} e^{i \nu^{\prime} t}+i \sqrt{\gamma} f^{\mathrm{in}}(t-s)^{\dagger} e^{i \nu^{\prime} t}\right] \\
\times\left[-i \eta \Omega \cos \theta_{L}+\eta g_{1} \sin \phi_{1} \cos \theta_{c} \widetilde{a}_{1}(t) e^{i \nu^{\prime} t}+\eta g_{2} \sin \phi_{2} \cos \theta_{c} \widetilde{a}_{2}(t) e^{-i \nu^{\prime} t}\right]-\kappa_{b} \widetilde{b}(t)+\sqrt{2 \kappa_{b}} \widetilde{b^{\mathrm{in}}}(t),
\end{gathered}
$$

where we have introduced the noise operators $\tilde{a}_{1}^{\text {in }}(t)$ $\equiv e^{-i \nu^{\prime} t} a_{1}^{\text {in }}(t), \widetilde{a}_{2}^{\text {in }}(t) \equiv e^{i \nu^{\prime} t} a_{2}^{\text {in }}(t)$, and $\widetilde{b}^{\text {in }}(t) \equiv e^{i \nu^{\prime} t} b^{\text {in }}(t)$, which are still $\delta$-correlated.

We insert in these equations the explicit expression for $B(t-s)$, thereby neglecting the terms oscillating at $\nu^{\prime}$ or faster. We finally perform the time integrals by making the Markovian approximation $\exp \left\{-\left(\gamma / 2 \pm i \Delta+i m \nu^{\prime}\right) s\right\}$ $\approx \delta(s) /\left(\gamma / 2 \pm i \Delta+i m \nu^{\prime}\right)$, for $m=-1,0,1$. After long, but straightforward calculations we get the final, effective QLE at leading order in the Lamb-Dicke parameter, which read

$$
\begin{gathered}
\dot{\tilde{a}}_{1}^{\dagger}(t)=i\left(\nu^{\prime}-\delta_{1}\right) \widetilde{a}_{1}^{\dagger}(t)+\chi_{1}^{*} \widetilde{b}(t)-\left(\kappa_{1}+\kappa_{1 L}-i \delta_{1 L}\right) \widetilde{a}_{1}^{\dagger}(t)+\sqrt{2 \kappa_{1}} \widetilde{a}_{1}^{\mathrm{in}}(t)^{\dagger}+\sqrt{2} \bar{\kappa}_{1 L} \widetilde{a}_{1 L}^{\text {in }}(t)^{\dagger}+F_{1}, \\
\dot{\tilde{a}}_{2}(t)=i\left(\nu^{\prime}+\delta_{2}\right) \widetilde{a}_{2}(t)+\chi_{2} \widetilde{b}(t)-\left(\kappa_{2}+\kappa_{2 L}+i \delta_{2 L}\right) \widetilde{a}_{2}(t)+\sqrt{2 \kappa_{2}} \widetilde{a}_{2}^{\text {in }}(t)+\sqrt{2} \bar{\kappa}_{2 L} \widetilde{a}_{2 L}^{\text {in }}(t)+F_{2}, \\
\dot{\tilde{b}}(t)=i\left(\nu^{\prime}-\nu\right) \widetilde{b}(t)+\bar{\chi}_{1} \widetilde{a}_{1}^{\dagger}(t)-\bar{\chi}_{2}^{*} \widetilde{a}_{2}(t)-\left(\kappa_{b}+\kappa_{2 b}-\kappa_{1 b}+i \delta_{b}\right) \widetilde{b}(t)+\sqrt{2 \kappa_{b}} \widetilde{b}^{\text {in }}(t)+\sqrt{2} \bar{\kappa}_{2 b} \widetilde{a}_{2 L}^{\text {in }}(t)-\sqrt{2} \bar{\kappa}_{1 b} \widetilde{a}_{1 L}^{\text {in }}(t)^{\dagger}+F_{b} .
\end{gathered}
$$

Let us now discuss each term appearing in the equations. The coupling coefficients are given by

$$
\begin{aligned}
& \chi_{1}=\eta \Omega g_{1}^{*} \cos \phi_{1}\left(\frac{\cos \theta_{L}}{\Delta-\nu^{\prime}+i \gamma / 2}+\frac{i \tan \phi_{1} \cos \theta_{c}}{\Delta+i \gamma / 2}\right), \\
& \chi_{2}=\eta \Omega g_{2}^{*} \cos \phi_{2}\left(\frac{\cos \theta_{L}}{\Delta+\nu^{\prime}+i \gamma / 2}+\frac{i \tan \phi_{2} \cos \theta_{c}}{\Delta+i \gamma / 2}\right), \\
& \bar{\chi}_{1}=\eta \Omega g_{1}^{*} \cos \phi_{1}\left(\frac{\cos \theta_{L}}{\Delta-\nu^{\prime}-i \gamma / 2}+\frac{i \tan \phi_{1} \cos \theta_{c}}{\Delta+i \gamma / 2}\right), \\
& \bar{\chi}_{2}=\eta \Omega g_{2}^{*} \cos \phi_{2}\left(\frac{\cos \theta_{L}}{\Delta+\nu^{\prime}-i \gamma / 2}+\frac{i \tan \phi_{2} \cos \theta_{c}}{\Delta+i \gamma / 2}\right),
\end{aligned}
$$

and correspond to the Raman processes, in which laser photons are scattered into the cavity mode with a change in the center-of-mass excitation, see Sec. IIIB2.

New fluctuation-dissipation sources appear in the equations. We first discuss noise terms appearing in Eqs. (54) and (55). In addition to cavity decay with rates $\kappa_{j}$ we find pro- cesses described by the decay terms with rate $\kappa_{1 L}$ and $\kappa_{2 L}$, and the corresponding Langevin noises $\tilde{a}_{1 L}^{\text {in }}(t)$ and $\tilde{a}_{2 L}^{\text {in }}(t)$, where

$$
\begin{gathered}
\kappa_{1 L}=\frac{\gamma}{2} \frac{\left|g_{1}\right|^{2} \cos ^{2} \phi_{1}}{\gamma^{2} / 4+\left(\Delta-\nu^{\prime}\right)^{2}}, \\
\kappa_{2 L}=\frac{\gamma}{2} \frac{\left|g_{2}\right|^{2} \cos ^{2} \phi_{2}}{\gamma^{2} / 4+\left(\Delta+\nu^{\prime}\right)^{2}}
\end{gathered}
$$

and

$$
\begin{aligned}
& \tilde{a}_{1 L}^{\mathrm{in}}(t)=f^{\mathrm{in}}(t) e^{-i \nu^{\prime} t}, \\
& \tilde{a}_{2 L}^{\mathrm{in}}(t)=f^{\mathrm{in}}(t) e^{i \nu^{\prime} t} .
\end{aligned}
$$

These noises describe input-output processes between the cavity modes and external modes, mediated by the atom. They possess the same correlation functions of the spontaneous emission noise $f^{\mathrm{in}}(t)$, and at the time scales of interest, $\nu^{\prime} t \gg 1$, they are uncorrelated from each other, thanks to the oscillating factors. Note that

$$
\bar{\kappa}_{1 L}=-i \sqrt{\frac{\gamma}{2}} \frac{g_{1} \cos \phi_{1}}{\gamma / 2+i\left(\Delta-\nu^{\prime}\right)},
$$




$$
\bar{\kappa}_{2 L}=i \sqrt{\frac{\gamma}{2}} \frac{g_{2}^{*} \cos \phi_{2}}{\gamma / 2-i\left(\Delta+\nu^{\prime}\right)},
$$

with $\kappa_{j L}=\left|\bar{\kappa}_{j L}\right|^{2}$. They originate from the scattering processes in which cavity photons are lost because they are absorbed and then spontaneously emitted by the atom, as has been discussed in Sec. III B 3.

The noise and dissipation terms in Eq. (56), in addition to the noise terms of the trap, are described by the decay terms with rate $\kappa_{1 b}$ and $\kappa_{2 b}$, and the corresponding Langevin noise operators $\tilde{a}_{1 L}^{\text {in }}(t)$ and $\tilde{a}_{2 L}^{\text {in }}(t)$. These processes originate from incoherent emission or absorption of a vibrational quantum accompanied by absorption and subsequent spontaneous emission of a laser photon. The emission of vibrational quanta takes place at rate

$$
\kappa_{2 b}=\frac{\gamma}{2} \frac{\eta^{2}|\Omega|^{2} \cos ^{2} \theta_{L}}{\gamma^{2} / 4+\left(\Delta+\nu^{\prime}\right)^{2}},
$$

while the rate of incoherent absorption of vibrational quanta is given by

$$
\kappa_{1 b}=\frac{\gamma}{2} \frac{\eta^{2}|\Omega|^{2} \cos ^{2} \theta_{L}}{\gamma^{2} / 4+\left(\Delta-\nu^{\prime}\right)^{2}} .
$$

In particular, when $\Delta<0$ then $\kappa_{2 b}>\kappa_{1 b}$ and the motion is cooled. Moreover,

$$
\begin{gathered}
\bar{\kappa}_{1 b}=\sqrt{\frac{\gamma}{2}} \frac{\eta \Omega \cos \theta_{L}}{\gamma / 2+i\left(\Delta-\nu^{\prime}\right)}, \\
\bar{\kappa}_{2 b}=\sqrt{\frac{\gamma}{2}} \frac{\eta \Omega^{*} \cos \theta_{L}}{\gamma / 2-i\left(\Delta+\nu^{\prime}\right)},
\end{gathered}
$$

with $\kappa_{j b}=\left|\bar{\kappa}_{j b}\right|^{2}$. If we consider the dynamics described by these terms only, these incoherent phonon absorption and emission processes lead to thermalization of the atomic motion at rate $\kappa_{2 b}-\kappa_{1 b}$, to a final effective mean vibrational number $n_{\text {th }}=\kappa_{1 b} /\left(\kappa_{2 b}-\kappa_{1 b}\right) \simeq|\Delta| / 4 \nu^{\prime}$, as in standard cooling [48]. However, the noise associated with these incoherent phonon absorptions and emissions is correlated with the noise terms $\widetilde{a}_{1 L}^{\text {in }}(t)$ and $\tilde{a}_{2 L}^{\text {in }}(t)$ describing scattering of cavity photons, because all these processes ultimately originate from spontaneous emission. This is why the noise terms in the Langevin equation for the atomic motion are directly expressed in terms of $\tilde{a}_{1 L}^{\text {in }}(t)$ and $\tilde{a}_{2 L}^{\text {in }}(t)$, making therefore this correlation evident.

The operators $F_{j}$ in Eqs. (49)-(53) represent nonlinear terms, which describe the noise associated with the incoherent part of the scattering processes discussed in Sec. IIIB2. These terms can be neglected with respect to the coherent processes, provided that $\gamma \ll|\Delta|$ and $\gamma \ll \nu$. In particular, the second inequality ensures that rates $\chi_{1}$ and $\chi_{2}$ differ appreciably, such that entanglement between the cavity modes can be established in a finite time [26]. We will focus on this regime, $\gamma \ll \nu$, in which we can thus neglect $F_{j}$ in the effective QLE when evaluating the spectrum of squeezing.

Finally, the frequency shifts of the two cavity modes and of the vibrational motion read

$$
\begin{gathered}
\delta_{1 L}=\frac{\left(\Delta-\nu^{\prime}\right)\left|g_{1}\right|^{2} \cos ^{2} \phi_{1}}{\gamma^{2} / 4+\left(\Delta-\nu^{\prime}\right)^{2}}, \\
\delta_{2 L}=\frac{\left(\Delta+\nu^{\prime}\right)\left|g_{2}\right|^{2} \cos ^{2} \phi_{2}}{\gamma^{2} / 4+\left(\Delta+\nu^{\prime}\right)^{2}}, \\
\delta_{b}=\frac{2 \Delta \eta^{2}|\Omega|^{2} \cos ^{2} \theta_{L}\left(\gamma^{2} / 4+\Delta^{2}-\nu^{\prime 2}\right)}{\left(\gamma^{2} / 4+\Delta^{2}-\nu^{\prime 2}\right)^{2}+\nu^{\prime 2} \gamma^{2}} \\
-\eta^{2}|\Omega|^{2} \cos ^{2} \theta_{L} \frac{\Delta}{\Delta^{2}+\gamma^{2} / 4}
\end{gathered}
$$

and from their form one can recognize the ac-Stark shifts reported in Sec. III B 4, with $\delta \omega_{j}=\delta_{j L} a_{j}^{\dagger} a_{j}$, Eq. (32), and $\delta \nu_{b}=\delta_{b} b^{\dagger} b$, Eq. (31), where now $\nu \rightarrow \nu^{\prime}$. Note that we have omitted a nonlinear shift at second order in the Lamb-Dicke parameter, which affects both cavity modes and motion. As discussed in Sec. 3.2.4, this is a small correction to $\delta_{j L}$, as it scales with $\eta^{2}$, while it may have a relevant effect on the center-of-mass dynamics. It can be neglected in the limit $\Omega \cos ^{2} \theta_{L} \gg g_{j} \cos ^{2} \theta_{c}$. Under this assumption, which we will consider in the rest of this manuscript, the spectrum of the center-of-mass is the spectrum of a harmonic oscillator, characterized by equidistant energy levels.

As the dynamics we seek relies on resonant interaction between the cavity modes and the vibrational motion, the two cavity modes should be exactly at resonance with the sidebands of the driving laser. Equation (73) provides an implicit equation for the actual vibrational angular frequency $\nu^{\prime}$. In the parameter regime $\eta|\Omega| \ll|\Delta|, \nu$ we find with good approximation

$$
\begin{aligned}
\nu^{\prime} \approx & \nu+\frac{2 \Delta \eta^{2}|\Omega|^{2} \cos ^{2} \theta_{L}\left(\gamma^{2} / 4+\Delta^{2}-\nu^{2}\right)}{\left(\gamma^{2} / 4+\Delta^{2}-\nu^{2}\right)^{2}+\nu^{2} \gamma^{2}} \\
& -\eta^{2}|\Omega|^{2} \cos ^{2} \theta_{L} \frac{\Delta}{\Delta^{2}+\gamma^{2} / 4}
\end{aligned}
$$

Taking also into account the frequency shifts of Eqs. (71) and (72), the resonance conditions are finally

$$
\begin{aligned}
& \delta_{1}=\delta_{1 L}+\nu^{\prime}, \\
& \delta_{2}=\delta_{2 L}-\nu^{\prime} .
\end{aligned}
$$

In the parameter regime $\gamma \ll \nu$, using conditions (75) and (76), we arrive therefore to the final QLE, describing the coherent interaction between the two cavity modes and the vibrational motion, competing with losses and noise processes due to spontaneous emission, cavity decay, and vibrational heating,

$$
\begin{aligned}
& \dot{\tilde{a}}_{1}^{\dagger}(t)=\chi_{1}^{*} \tilde{b}(t)-\left(\kappa_{1}+\kappa_{1 L}\right) \tilde{a}_{1}^{\dagger}(t)+\sqrt{2 \kappa_{1}} \widetilde{a}_{1}^{\text {in }}(t)^{\dagger}+\sqrt{2} \bar{\kappa}_{1 L} \widetilde{a}_{1 L}^{\text {in }}(t)^{\dagger}, \\
& \dot{\tilde{a}}_{2}(t)=\chi_{2} \tilde{b}(t)-\left(\kappa_{2}+\kappa_{2 L}\right) \tilde{a}_{2}(t)+\sqrt{2 \kappa_{2}} \widetilde{a}_{2}^{\text {in }}(t)+\sqrt{2} \bar{\kappa}_{2 L} \widetilde{a}_{2 L}^{\text {in }}(t),
\end{aligned}
$$




$$
\begin{aligned}
\dot{\tilde{b}}(t)= & \bar{\chi}_{1} \widetilde{a}_{1}^{\dagger}(t)-\bar{\chi}_{2}^{*} \widetilde{a}_{2}(t)-\left(\kappa_{b}+\kappa_{2 b}-\kappa_{1 b}\right) \widetilde{b}(t)+\sqrt{2 \kappa_{b}} \widetilde{b}^{\text {in }}(t) \\
& +\sqrt{2} \bar{\kappa}_{2 b} \tilde{a}_{2 L}^{\text {in }}(t)-\sqrt{2} \bar{\kappa}_{1 b} \widetilde{a}_{1 L}^{\text {in }}(t)^{\dagger} .
\end{aligned}
$$

\section{B. Evaluation of the spectrum of squeezing}

We now use Eqs. (77)-(79) in order to determine the stationary spectrum of squeezing of the light at the cavity output. We consider

$$
\begin{gathered}
I_{-}^{\text {out }}(t)=a_{1}^{\text {out }}(t)+a_{1}^{\text {out }}(t)^{\dagger}-a_{2}^{\text {out }}(t)-a_{2}^{\text {out }}(t)^{\dagger}, \\
I_{+}^{\text {out }}(t)=-i\left[a_{1}^{\text {out }}(t)-a_{1}^{\text {out }}(t)^{\dagger}+a_{2}^{\text {out }}(t)-a_{2}^{\text {out }}(t)^{\dagger}\right],
\end{gathered}
$$

corresponding respectively to the difference between the amplitude quadratures, and the sum of the phase quadratures of the two sideband modes. These are the quadratures exhibiting two-mode squeezing in the case of pulsed excitation in this setup, see Refs. $[25,26]$. The output cavity fields $a_{j}^{\text {out }}(t)$ in Eqs. (80) and (81) are given by the usual input-output relation

$$
a_{j}^{\text {out }}(t)=\sqrt{2 \kappa_{j}} a_{j}(t)-a_{j}^{\text {in }}(t), \quad j=1,2 .
$$

The spectrum of squeezing can be calculated by evaluating the Fourier transforms

$$
\hat{I}_{ \pm}^{\text {out }}(\omega)=\int d t e^{i \omega t} I_{ \pm}^{\text {out }}(t),
$$

and using the fact that at the stationary state it is

$$
\left\langle\hat{I}_{ \pm}^{\text {out }}(\omega) \hat{I}_{ \pm}^{\text {out }}\left(\omega^{\prime}\right)+\hat{I}_{ \pm}^{\text {out }}\left(\omega^{\prime}\right) \hat{I}_{ \pm}^{\text {out }}(\omega)\right\rangle=8 \pi S_{ \pm}(\omega) \delta\left(\omega+\omega^{\prime}\right),
$$

where we have normalized the spectrum so that the shot noise level corresponds to $S_{ \pm}(\omega)=1$. Two-mode squeezing is found when one spectrum of squeezing takes values below the shot noise limit at some $\omega$. From the Fourier transform of Eqs. (77)-(79) one can see that $S_{+}(\omega)=S_{-}(\omega) \equiv S(\omega)$, which implies that in the present case two-mode squeezing is equivalent to EPR-like entanglement between the two output cavity modes. This is easily verified by applying a sufficient criterion for entanglement, such as the "sum" criterion of Duan et al. [52], or the product criterion of Refs. [53,54]. With the chosen normalization for the output cavity modes at $\omega$, the sum criterion reads

$$
S_{+}(\omega)+S_{-}(\omega)<2,
$$

while the product criterion gives

$$
S_{+}(\omega) S_{-}(\omega)<1,
$$

so that in our case both criteria imply that the two output modes are EPR-like entangled as soon as $S(\omega)<1$. The squeezing spectrum $S(\omega)$ can be obtained from the Fourier transform of the Langevin equations after long but straightforward algebra, yielding a cumbersome expression which will not be reported here. This expression becomes considerably simpler in the limit $|\Omega|,\left|g_{j}\right|, \gamma \ll|\Delta|$ and $\eta \ll 1$. In this limit the additional loss processes due to spontaneous emis- sion, associated with the rates $\kappa_{j L}$ and $\kappa_{j b}(j=1,2)$, are typically negligible, that is, $\kappa_{j L}, \kappa_{j b} \ll \kappa$. Moreover, we consider the case of ion traps, where heating of the atomic motion is negligible with respect to all radiative noise sources [55]. Finally, as the two cavity modes are very close in frequency, they will have very similar properties, in particular we can take $\kappa_{1}=\kappa_{2}=\kappa$. In this parameter regime the main aspects of the squeezing spectrum can be grasped from its analytical expression. One finds

$$
S(\omega)=1-\frac{\kappa^{2}\left(\Theta^{4}-\Sigma^{4}\right)}{\left(\kappa^{2}+\omega^{2}\right)\left[\left(\omega^{2}-\Theta^{2}\right)^{2}+\omega^{2} \kappa^{2}\right]},
$$

where $\Theta=\sqrt{\left|\chi_{2}\right|^{2}-\left|\chi_{1}\right|^{2}}$ as given in Eq. (2),

$$
\Sigma=\sqrt{\left.|| \chi_{2}\right|^{2}+\left|\chi_{1}\right|^{2}-2 \chi_{1} \chi_{2} \mid},
$$

and we have used that $\chi_{j}=\bar{\chi}_{j}$ when $\gamma \ll|\Delta|$ [see Eqs. (57)-(60)]. Note that due to the transformations which we have applied, the results which appear around $\omega=0$ in $S(\omega)$ describe quantum correlations of noise components in the optical signal at $\omega_{L}-\nu^{\prime} \pm \omega$ with those at $\omega_{L}+\nu^{\prime} \pm \omega$, i.e. correlated fluctuations of the two modes at the same offset from their center frequencies.

From Eq. (87) one notes that the properties of the spectrum are mainly determined by the ratio $\Theta / \kappa$. In fact, the denominator in Eq. (87) has always three poles in the lower complex half-plane, one which is always imaginary at $\omega_{0}=-i \kappa$, and two poles at $\omega_{ \pm}=-i \kappa / 2 \pm \sqrt{\Theta^{2}-\kappa^{2} / 4}$. Therefore, when $\Theta / \kappa \gg 1$ the two poles at $\omega_{ \pm}$have a nonzero real part and the spectrum is characterized by three wellseparated inverted Lorentzian peaks, one at $\omega=0$ with a full width at half maximum (FWHM) of $2 \kappa$ and the other two symmetrically placed at $\omega \approx \pm \Theta$, with FWHM $\kappa$. At the center of these peaks one has $S=(\Sigma / \Theta)^{4} \simeq\left[\left(\left|\chi_{2}\right|-\left|\chi_{1}\right|\right) /\left(\left|\chi_{2}\right|\right.\right.$ $\left.\left.+\left|\chi_{1}\right|\right)\right]^{2}$, approaching $S(\omega)=0$, i.e., infinite two-mode squeezing, for $\left|\chi_{2}\right| \simeq\left|\chi_{1}\right|$. Therefore, when $\Theta>\kappa$ we find two-mode squeezing within three narrow bandwidths around $\omega=0$ and $\omega= \pm \Theta$. In the opposite case of $\Theta \leqslant \kappa / 2$, the three poles are all on the imaginary axis, and the spectrum shows only one inverted Lorentzian peak at $\omega=0$. When $\Theta / \kappa \ll 1$, this peak becomes very narrow, with FWHM $\sim 2 \Theta^{2} / \kappa$.

It is remarkable that even for $\kappa>\Theta$ one finds almost perfect squeezing in the difference of amplitude quadratures at $\omega=0$. This can be understood, as in the regime we consider the scattered photons due to spontaneous emission are negligible with respect to those lost through the output cavity mirror $\left(\kappa_{j L}, \kappa_{j b} \ll \kappa\right)$. This implies that most of the intracavity photons are detected at the output. These photons are almost perfectly correlated at $\omega=0$ and therefore would give $S(0)$ $\simeq 0$. In this regime, a large cavity decay rate $\kappa$ has only the effect of narrowing the squeezing bandwidth. On the contrary, if the photon scattering by spontaneous emission is no more negligible, two-mode squeezing soon degrades, even at $\omega=0$ (see, for example, Ref. [32]).

The presence of the three-pole structure in the squeezing spectrum is unique with respect to the spectral features usually encountered in the parametric oscillator (either below and above threshold, see, e.g., Ref. [32]). This structure is due to the coherent interaction of the two cavity modes with 
the quantized atomic motion, i.e., it arises from the coherent microscopic processes underlying the dynamic establishment of quantum correlations. The peculiar spectral properties can be exploited to achieve optimal broadband two-mode squeezing when the three peaks merge, which happens when $\Theta=\kappa$. In this case one easily sees from Eq. (87) that

$$
S(\omega)=1-\frac{\kappa^{2}\left(\Theta^{4}-\Sigma^{4}\right)}{\Theta^{6}+\omega^{6}},
$$

i.e., one has large, uniform squeezing for a wide bandwidth of frequencies. The fact that $\Theta=\kappa$ is the condition for the best two-mode squeezing in the output can be easily understood noticing that $\Theta$ is the angular frequency at which twomode squeezing inside the cavity is periodically built up (see Refs. [25,26]). Therefore, when $\Theta=\kappa$, squeezing is generated inside the cavity at the same rate at which it is transferred to the output field. In contrast, in the other two cases, squeezing is not efficiently generated in the output because either the output coupling happens too fast, i.e., before full intracavity squeezing is established, or the output coupling is sufficiently slow to allow that energy is stored inside the cavity and the two-mode squeezing is coherently reconverted into independent states before it is coupled out.

\section{Results}

We now consider the exact squeezing spectrum $S(\omega)$, defined in Eq. (84), where the operator (80) is evaluated using the output relation (82), such that the operators $a_{j}(t), a_{j}^{\dagger}(t)$ are the solutions of the QLEs (77)-(79) including all noise and loss terms.

The parameter regime we consider has been discussed in detail in Ref. [26]. We take a $F=0 \leftrightarrow F^{\prime}=1$ atomic transition with the quantization axis $\vec{B}$ along the cavity axis, and $\vec{B}, \vec{k}_{L}$, and laser polarization $\vec{E}_{L}$ mutually orthogonal. Relation $\gamma$ $\ll \nu$ can be fulfilled by the intercombination line of an Indium ion at $\gamma=2 \pi \times 360 \mathrm{kHz}$ in a trap at $\nu=2 \pi \times 3 \mathrm{MHz}$, for which $\eta \simeq 0.1$. We consider a geometrical configuration corresponding to $\theta_{L}=0, \theta_{c}=\pi / 2$, and $\phi_{1}=\phi_{2}=0$, which means that the ion motion takes place along the direction of the laser beam and orthogonally to the cavity axis, and that the trap center coincides with an antinode of the cavity modes. In such a case, the coupling coefficients of Eqs. (57)-(60) are determined by the first term only. Moreover in this case $\Omega \cos ^{2} \theta_{L} \gg g_{j} \cos ^{2} \theta_{c} \simeq 0$, and therefore, as discussed in Secs. III B 4 and IV A, the small Stark-shift correction to $\delta_{j L}$, scaling with $\eta^{2}$ can be neglected. If we consider that the ion couples to two non-degenerate polarization modes of a resonator with vacuum Rabi couplings $g \approx 2 \pi$ $\times 0.6 \mathrm{MHz}$, and we take laser Rabi frequency $\Omega=2 \pi$ $\times 18 \mathrm{MHz}$ and detuning $\Delta=2 \pi \times 60 \mathrm{MHz}$, we obtain $\Theta / 2 \pi \simeq 7.9 \mathrm{kHz}$, see Ref. [26]. The condition $\Theta \gg \kappa$ is found for a finesse $\mathcal{F} \simeq 10^{6}$ and free spectral range $\delta \omega=2 \pi$ $\times 1 \mathrm{GHz}$, so that $\kappa=2 \pi \times 1 \mathrm{KHz}$. The corresponding spectrum of squeezing is displayed in Fig. 3 (full line). It exhibits three minima at $\omega=0, \pm \Theta$, which correspond to three separated regions of narrow-band squeezing. The two bands around $\omega= \pm \Theta$ have width $\kappa$, while the central one has width

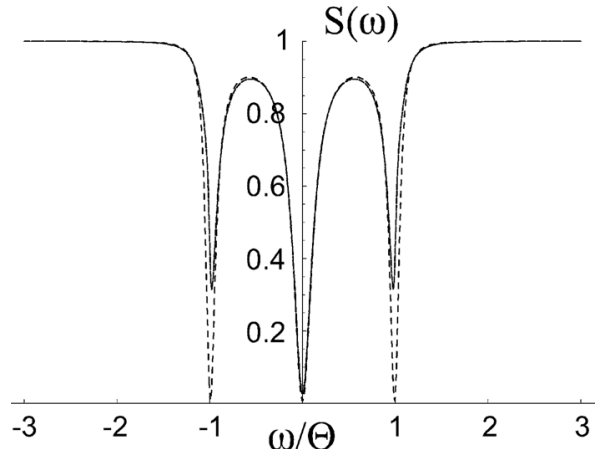

FIG. 3. Squeezing spectrum $S(\omega)$ as a function of the sideband frequency $\omega$ (in units of $\Theta$ ) when $\Theta \simeq 8 \kappa$. Its behavior is well reproduced by the approximate analytical expression of Eq. (87) (dashed line). The parameters are discussed in the text.

$2 \kappa$ and shows almost $100 \%$ squeezing. These features are well reproduced by the analytical expression (87) (see dashed line in Fig. 3), except that the latter predicts very large squeezing also for the peaks at $\omega= \pm \Theta$. The success of the simplified expression (87) is due to the fact that, with the chosen parameter values, the loss rates due to the various scattering processes are at least ten times smaller than the cavity output loss rates $\kappa_{1}=\kappa_{2}=\kappa$, and therefore do not have a relevant effect on the spectrum. We have also considered a realistic ion vibrational heating rate $\kappa_{h}=\kappa_{b} \bar{N}=2 \pi \times 0.1 \mathrm{kHz}$, which, however, gives an effect which is negligible even with respect to that due to photon scattering. The appearance of three minima is a novel behavior to our knowledge, and it arises from the coherent microscopic dynamics, as $\Theta$ modulates the exchange of excitations and correlations between the cavity modes and the center-of-mass motion.

The most interesting regime of broadband two-mode squeezing, when $\Theta \sim \kappa$, is shown in Fig. 4, which displays the squeezing spectrum in the case of the same parameter values of Fig. 3 except for a lower cavity finesse $\mathcal{F} \simeq 10^{5}$, implying $\kappa=2 \pi \times 10 \mathrm{kHz}$. The three minima merge into a single broad one, centered around $\omega=0$, whose width is determined by $\kappa=\Theta$. Also in this case one gets almost perfect squeezing at the center, and these features are well reproduced by the simple analytical expression of Eq. (89) (dashed line in Fig. 4).

Finally, in Fig. 5 we consider the case $\kappa>\Theta$. We have still kept the parameter values of Fig. 3, but we have now

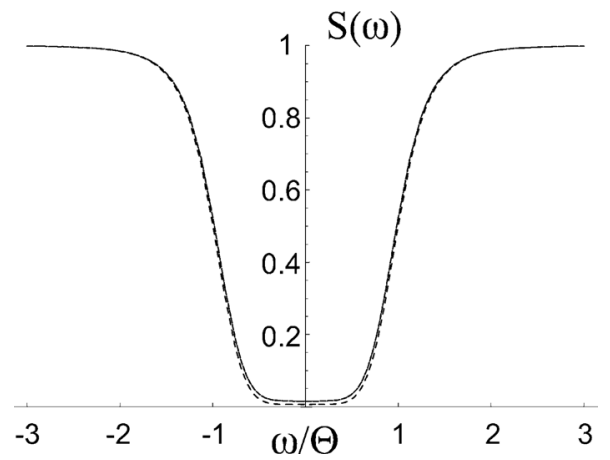

FIG. 4. Squeezing spectrum $S(\omega)$ when $\Theta=\kappa$. Its behavior is well reproduced by the approximate analytical expression of Eq. (87) (dashed line). The parameters are discussed in the text. 


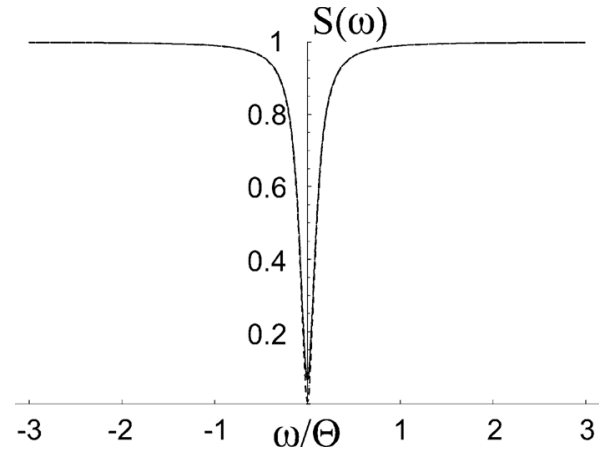

FIG. 5. Squeezing spectrum $S(\omega)$ when $\Theta \simeq \kappa / 10$. Its behavior is well reproduced by the approximate analytical expression of Eq. (87) (dashed line). The parameters are discussed in the text.

considered a cavity with finesse $\mathcal{F} \sim 10^{4}$, implying $\kappa=2 \pi$ $\times 100 \mathrm{kHz}$. The squeezing features are visibly worsened, as in this regime losses are faster than the typical time scale in which correlations between the field modes are established. One has still two-mode squeezing around $\omega=0$, but with a very narrow bandwidth which is roughly given by $\Theta^{2} / 2 \kappa$.

\section{CONCLUSIONS}

The resonance fluorescence of a confined, single, laserdriven atom exhibits EPR entanglement, or two-mode squeezing, in the field modes which interact resonantly with the Stokes and anti-Stokes transitions created by the atomic motion. By coupling these sidebands to a high-finesse optical cavity, we have shown how to create continuous-wave (cw) two-mode squeezed light output from the cavity. At the microscopic level, the process is based on the mechanical effect of light, which allows for quantum-coherent generation and control of entanglement between the motion and the cavity modes. The scattering processes have been characterized and described in simple physical pictures using scattering matrix theory, and the squeezing spectrum has been calculated using quantum Langevin equations.
Peculiar spectral properties are predicted for the squeezing spectrum of the cavity output. They may be divided into three regimes of the cavity output rate $\kappa$ relative to the frequency $\Theta$ of creation of two-mode squeezing inside the cavity. The squeezing spectrum can consist of a single peak $(\kappa>\Theta)$, three peaks $(\kappa<\Theta)$, or one broad, homogeneous band $(\kappa=\Theta)$. Simple analytical approximations have been derived for the three relevant regimes.

The squeezing spectrum in the different parameter regimes has been calculated for an experimentally accessible case of a single trapped ion as a specific example. The results for this specific system show all the features predicted by the general derivations, exhibiting spectral properties of twomode squeezing which are unique when compared with conventional Optical-parameter-amplifier-type of sources.

In particular, starting from the most fundamental individual quantum systems, a single atom and an optical cavity, we have designed a nonlinear optical source. This is therefore a paradigmatic model system exhibiting the connection between microscopic, quantum-coherent dynamics and macroscopic nonlinear device properties. Its efficiency and the high-degree of control one can achieve on its dynamics offer promising perspectives for the realization of quantum light sources for quantum networking [56,57].

\section{ACKNOWLEDGMENTS}

The authors acknowledge stimulating discussions with and helpful comments from P. Cañizares, S. Mancini, and P. Tombesi. One of the authors (D.V.) acknowledges the Grup d'Optica at the Universitat Autonoma de Barcelona for hospitality during completion of this work. This work was partly supported by the European Commission (CONQUEST network, Grant No. MRTN-CT-2003-505089; SCALA, Contract No. 015714) and by the Spanish Ministerio de Educación y Ciencia (LACSMY, Grant No. FIS2004-05830; Ramon-yCajal individual fellowship; QLIQS, Grant No. FIS200508257).
[1] A. Kuzmich, W. P. Bowen, A. D. Boozer, A. Boca, C. W. Chou, L.-M. Duan, and H. J. Kimble, Nature (London) 423, 731 (2003).

[2] V. Josse, A. Dantan, L. Vernac, A. Bramati, M. Pinard, and E. Giacobino, Phys. Rev. Lett. 91, 103601 (2003); V. Josse, A. Dantan, A. Bramati, M. Pinard, and E. Giacobino, ibid. 92, 123601 (2004).

[3] C. H. van der Wal, M. D. Eisaman, A. André, R. L. Walsworth, D. F. Phillips, A. S. Zibrov, and M. D. Lukin, Science 301, 196 (2003).

[4] M. D. Lukin, Rev. Mod. Phys. 75, 457 (2003).

[5] B. Julsgaard, J. Sherson, J. I. Cirac, J. Fiurasek, and E. S. Polzik, Nature (London) 432, 482 (2004).

[6] T. Chanelière, D. N. Matsukevich, S. D. Jenkins, S.-Y. Lan, T. A. B. Kennedy, and A. Kuzmich, Nature (London) 438, 833 (2005)
[7] M. D. Eisaman, A. André, F. Massou, M. Fleischhauer, A. S. Zibrov, and M. D. Lukin, Nature (London) 438, 837 (2005).

[8] B. Julsgaard, A. Kozhekin, and E. S. Polzik, Nature (London) 413, 400 (2001).

[9] C. W. Chou, H. de Riedmatten, D. Felinto, S. V. Polyakov, S. J. van Enk, and H. J. Kimble, Nature (London) 438, 828 (2005).

[10] D. N. Matsukevich, T. Chanelière, S. D. Jenkins, S.-Y. Lan, T. A. B. Kennedy, and A. Kuzmich, Phys. Rev. Lett. 96, 030405 (2006).

[11] K. W. Chan, C. K. Law, and J. H. Eberly, Phys. Rev. Lett. 88, 100402 (2002); K. W. Chan, C. K. Law, and J. H. Eberly, Phys. Rev. A 68, 022110 (2003)

[12] B. Blinov, D. L. Moehring, L.-M. Duan, and C. Monroe, Nature (London) 428, 153 (2004).

[13] J. Volz, M. Weber, D. Schlenk, W. Rosenfeld, J. Vrana, K. 
Saucke, C. Kurtsiefer, and H. Weinfurter, Phys. Rev. Lett. 96, 030404 (2006).

[14] K. An, J. J. Childs, R. R. Dasari, and M. S. Feld, Phys. Rev. Lett. 73, 3375 (1994).

[15] J. McKeever, A. Boca, A. D. Boozer, J. R. Buck, and H. J. Kimble, Nature (London) 425, 268 (2003).

[16] A. Kuhn, M. Hennrich, and G. Rempe, Phys. Rev. Lett. 89, 067901 (2002); T. Legero, T. Wilk, M. Hennrich, G. Rempe, and A. Kuhn, ibid. 93, 070503 (2004).

[17] J. McKeever, A. Boca, A. D. Boozer, R. Miller, J. R. Buck, A. Kuzmich, and H. J. Kimble, Science 303, 1992 (2004).

[18] M. Keller, B. Lange, K. Hayasaka, W. Lange, and H. Walther, Nature (London) 431, 1075 (2004).

[19] B. Darquié, M. P. A. Jones, J. Dingjan, J. Beugnon, S. Bergamini, Y. Sortais, G. Messin, A. Browaeys, and P. Grangier, Science 309, 454 (2005).

[20] J. M. Raimond, M. Brune, and S. Haroche, Rev. Mod. Phys. 73, 565 (2001); B. T. H. Varcoe, S. Brattke, M. Weidinger, and H. Walther, Nature (London) 403, 743 (2000).

[21] D. Leibfried, R. Blatt, C. Monroe, and D. Wineland, Rev. Mod. Phys. 75, 281 (2003).

[22] See, for instance, D. Schrader, I. Dotsenko, M. Khudaverdyan, Y. Miroshnychenko, A. Rauschenbeutel, and D. Meschede, Phys. Rev. Lett. 93, 150501 (2004); S. Nußmann, M. Hijlkema, B. Weber, F. Rohde, G. Rempe, and A. Kuhn, ibid. 95, 173602 (2005).

[23] H. Zeng and F. Lin, Phys. Rev. A 50, R3589 (1994); A. S. Parkins and H. J. Kimble, J. Opt. Soc. Am. B 1, 496 (1999).

[24] A. Peng and A. S. Parkins, Phys. Rev. A 65, 062323 (2002).

[25] G. Morigi, J. Eschner, S. Mancini, and D. Vitali, Phys. Rev. Lett. 96, 023601 (2006).

[26] G. Morigi, J. Eschner, S. Mancini, and D. Vitali, Phys. Rev. A 73, 033822 (2006).

[27] M. Lindberg, Phys. Rev. A 34, 3178 (1986).

[28] J. I. Cirac, R. Blatt, A. S. Parkins, and P. Zoller, Phys. Rev. A 48, 2169 (1993).

[29] Ch. Raab, J. Eschner, J. Bolle, H. Oberst, F. Schmidt-Kaler, and R. Blatt, Phys. Rev. Lett. 85, 538 (2000).

[30] M. Bienert, W. Merkel, and G. Morigi, Phys. Rev. A 73, 033402 (2006).

[31] A. Einstein, B. Podolsky, and R. Rosen, Phys. Rev. 47, 777 1935.

[32] D. F. Walls and G. J. Milburn, Quantum Optics (Springer, Berlin, 1994).

[33] D. F. Walls and P. Zoller, Phys. Rev. Lett. 47, 709 (1981).

[34] L. Mandel, Phys. Rev. Lett. 49, 136 (1982).

[35] A. Aspect, G. Roger, S. Reynaud, J. Dalibard, and C. CohenTannoudji, Phys. Rev. Lett. 45, 617 (1980).

[36] C. A. Schrama, G. Nienhuis, H. A. Dijkerman, C. Steijsiger, and H. G. M. Heideman, Phys. Rev. A 45, 8045 (1992).

[37] G. Nienhuis, Phys. Rev. A 47, 510 (1993).

[38] S. Bali, F. A. Narducci, and L. Mandel, Phys. Rev. A 47, 5056 (1993).

[39] R. L. de Matos Filho and W. Vogel, Phys. Rev. A 49, 2812 (1994).

[40] M. Jakob and G. Y. Kryuchkyan, Phys. Rev. A 59, 2111 (1999).

[41] M. G. Raizen, L. A. Orozco, M. Xiao, T. L. Boyd, and H. J. Kimble, Phys. Rev. Lett. 59, 198 (1987).

[42] M. Jakob and J. Bergou, Phys. Rev. A 60, 4179 (1999).

[43] R. M. Serra, C. J. Villas-Boas, N. G. de Almeida, and M. H. Y. Moussa, Phys. Rev. A 71, 045802 (2005).

[44] J. Ph. Karr, A. Baas, E. Giacobino, Phys. Rev. A 69, 063807 (2004); A. Baas, J.-Ph. Karr, M. Romanelli, A. Bramati, and E. Giacobino, Phys. Rev. Lett. 96, 176401 (2006).

[45] S. Mancini, D. Vitali, and P. Tombesi, Phys. Rev. Lett. 90, 137901 (2003).

[46] S. Pirandola, S. Mancini, D. Vitali, and P. Tombesi, Phys. Rev. A 68, 062317 (2003).

[47] We remark that Eq. (13) is at first order in the Lamb-Dicke parameter, nevertheless we have included also the term $\eta^{2}\left(2 b^{\dagger} b+1\right)$, which belongs to the second-order expansion. This term gives rise to ac Stark shifts induced by the field, which should be systematically taken into account when one considers transition rates between vibrational states (which are at second order in $\eta$ ). See Sec. III B and Refs. [28,49].

[48] S. Stenholm, Rev. Mod. Phys. 58, 699 (1986).

[49] J. I. Cirac, M. Lewenstein, and P. Zoller, Phys. Rev. A 51, 1650 (1995).

[50] S. Zippilli and G. Morigi, Phys. Rev. Lett. 95, 143001 (2005); Phys. Rev. A 72, 053408 (2005).

[51] Here, pure heating corresponds to the limit $\kappa_{b} \rightarrow 0, \bar{N} \rightarrow \infty$, with $\kappa_{b} \bar{N} \equiv \kappa_{h}$, the heating rate, kept constant.

[52] L. M. Duan, G. Giedke, J. I. Cirac, and P. Zoller, Phys. Rev. Lett. 84, 2722 (2000).

[53] S. Mancini, V. Giovannetti, D. Vitali, and P. Tombesi, Phys. Rev. Lett. 88, 120401 (2002).

[54] V. Giovannetti, S. Mancini, D. Vitali, and P. Tombesi, Phys. Rev. A 67, 022320 (2003).

[55] Typical heating rates for the ionic motion in traps are of the order of $0.1 \mathrm{kHz}$, see for instance Q. A. Turchette, D. Kielpinski, B. E. King, D. Leibfried, D. M. Meekhof, C. J. Myatt, M. A. Rowe, C. A. Sackett, C. S. Wood, W. M. Itano, C. Monroe, and D. J. Wineland, Phys. Rev. A 61, 063418 (2000).

[56] J. I. Cirac, P. Zoller, H. J. Kimble, and H. Mabuchi, Phys. Rev. Lett. 78, 3221 (1997).

[57] B. Kraus and J. I. Cirac, Phys. Rev. Lett. 92, 013602 (2004). 